\title{
Materials communicating with the BIM: aims and first results of the McBIM project
}

\author{
William DERIGENT ${ }^{1}$, Michaël DAVID ${ }^{1}$, Hang WAN $^{1}$, Daniela \\ DRAGOMIRESCU ${ }^{2,4}$, Alex TAKACS ${ }^{2,3}$, Gaël LOUBET $^{2}$, Ana-Maria ROXIN ${ }^{5}$, \\ Rolland MELET ${ }^{6}$, Laurent MONTEGUT ${ }^{6}$ \\ ${ }^{1}$ CRAN CNRS UMR 7039, Université de Lorraine, Campus Sciences, 54506 Vandoeuvre-lès-Nancy \\ Cedex, France \\ ${ }^{2}$ Laboratory for Analysis and Architecture of Systems, National Center for Scientific Research, F-31031 \\ Toulouse, France \\ ${ }^{3}$ University Paul Sabatier, University of Toulouse, F-31062 Toulouse, France \\ ${ }^{4}$ National Institute of Applied Sciences of Toulouse, F-31077 Toulouse, France \\ ${ }^{5}$ Laboratoire d'Informatique de Bourgogne (LIB) - EA 7534, University of Bourgogne Franche-Comté, \\ Dijon, France \\ 6360SmartConnect/FINAO SAS, 19 Avenue de la Gare, 83720 Trans en Provence, France \\ Corresponding author: William DERIGENT (william.derigent@univ-lorraine.fr)
}

\section{Introduction}

The Internet of Things (IoT) is widely used in manufacturing, logistics or monitoring applications. The application of IoT makes the product more intelligent, its high flexibility brings new challenge to the Intelligent Manufacturing System (IMS). In 2009, the Research Center for Automatic Control (CRAN) began to study the "communicating materials" concept, which are materials ableto process, store data and communicate with the environment. During the related thesis of Kubler [1] and Mekki [2], this concept has been applied to construction industry.

The McBIM Project [3] (Material communicating with the BIM - Building Information Modelling) aims to design a "communicating concrete". Namely, it is the concrete equipped with embedded wireless micro-sensor network (WSN), which can measure the physical environment, store the information and exchange data with BIM platforms. Another objective is to demonstrate the usefulness of this new concept for different phases of a building's lifecycle, i.e. the manufacturing, construction and exploitation phases. This project is funded by the French National Research Agency and is coordinated by the CRAN with 2 other French laboratories (LAAS, LIB) and one company (360 SmartConnect/FINAO SAS). In this project, different partners focus on different areas. Where the CRAN works on the network and information management, the LAAS designs the sensing and communicating nodes, the LIB studies data interoperability and all these works are then implemented by 360 SmartConnect/FINAO SAS.

Section 2 introduces the McBIM project and the concept of communicating concrete, detailing its main constraints and behaviors for each phase of its lifecycle. As will be described, a communicating concrete is composed of physical components (i.e. the concrete structure, embedded WSN) and virtual components (i.e. database, applications). This paper mainly focuses on the physical parts and more specifically on the WSN embedded in the concrete. Indeed, section 3 is devoted to the development of a wireless and battery-free sensor node prototype and on first tests and results in the air. 
Section 4 introduces some existing solutions of data collection architectures for a network of wireless sensor nodes. A synthesis of those solutions and their use in our application case are then discussed. Last section concludes and gives some perspectives for the development of this work.

\section{Description of the McBIM project}

\subsection{Context and challenges}

The communicating concrete (see Figure 1) consists of many sensing and communicating nodes. The sensing nodes will periodically monitor the physical parameters (like temperature, humidity ...) of the concrete. Communicating nodes aggregate received data and transmit it to remote servers thanks to BIM standards. Besides, manufacturing data (like its physical properties or manufacturing actors) may also be considered. The main constraint is that the communicating concrete element has to function several decades from the manufacturing phase to the latest of the exploitation phase.
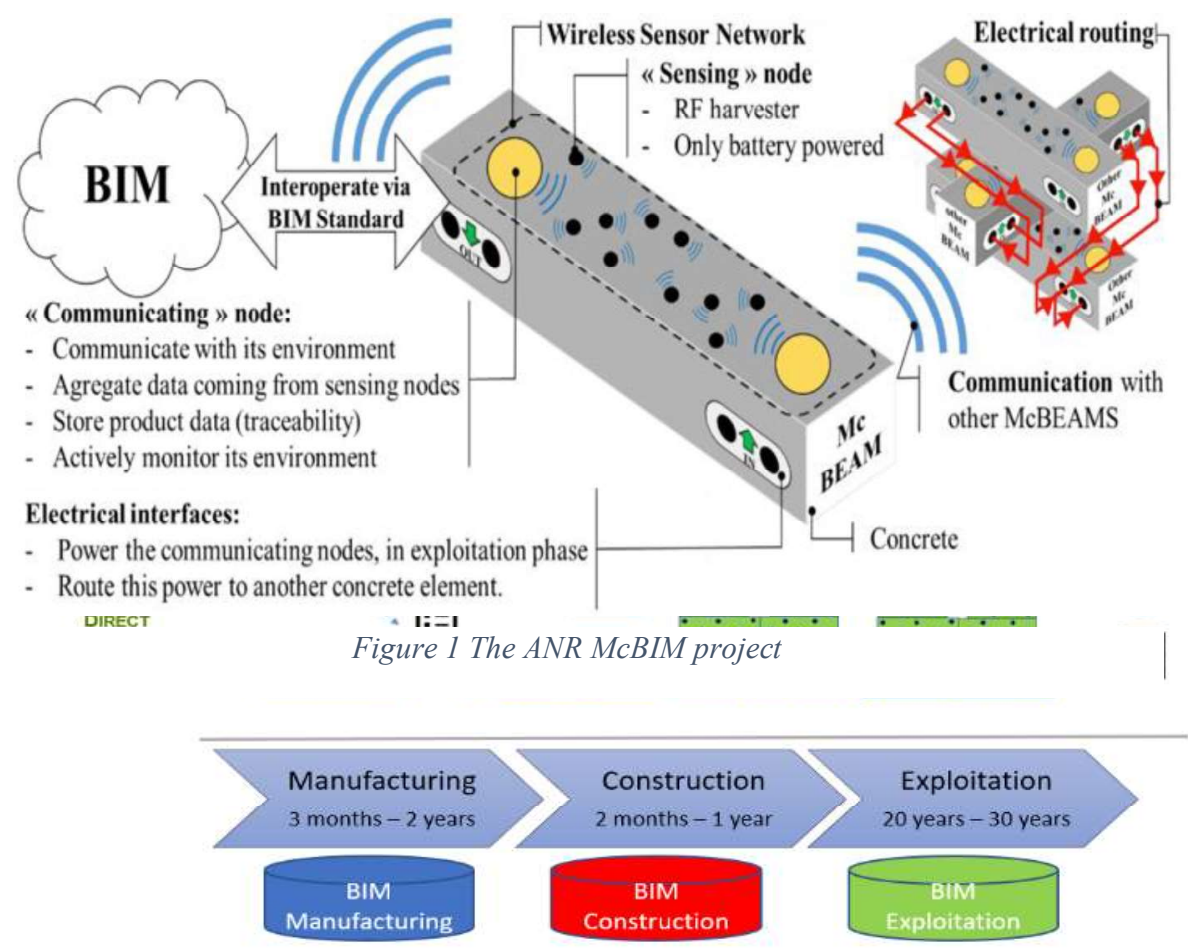

Figure 2 McBIM communicating concrete lifecycle

Besides, the communicating concrete behaviors are different along its lifecycle (see Figure 2). During the manufacturing phase, the WSN nodes are inserted and initialized. The communicating concretes periodically (by example every hour) monitor its physical status, stores the physical propriety information and manufacturing actor information. 
Those data are accessible directly via a reader device or remotely via the internet. For the construction phase, communicating concretes will be assembled together. In this case, as communicating concretes arrive, auto-organization is then needed to dynamically define a 3D network to achieve energy savings. Due to the high flexibility in this step, concrete has to report its status frequently (such as every half-hour) to ensure the safety of construction and updates the network information. When the construction is completed, the large 3D static WSN will regularly (by example every half-day) monitor struct health data (such as cracks, temperature, corrosion, etc.) to ensure the safety of the building.

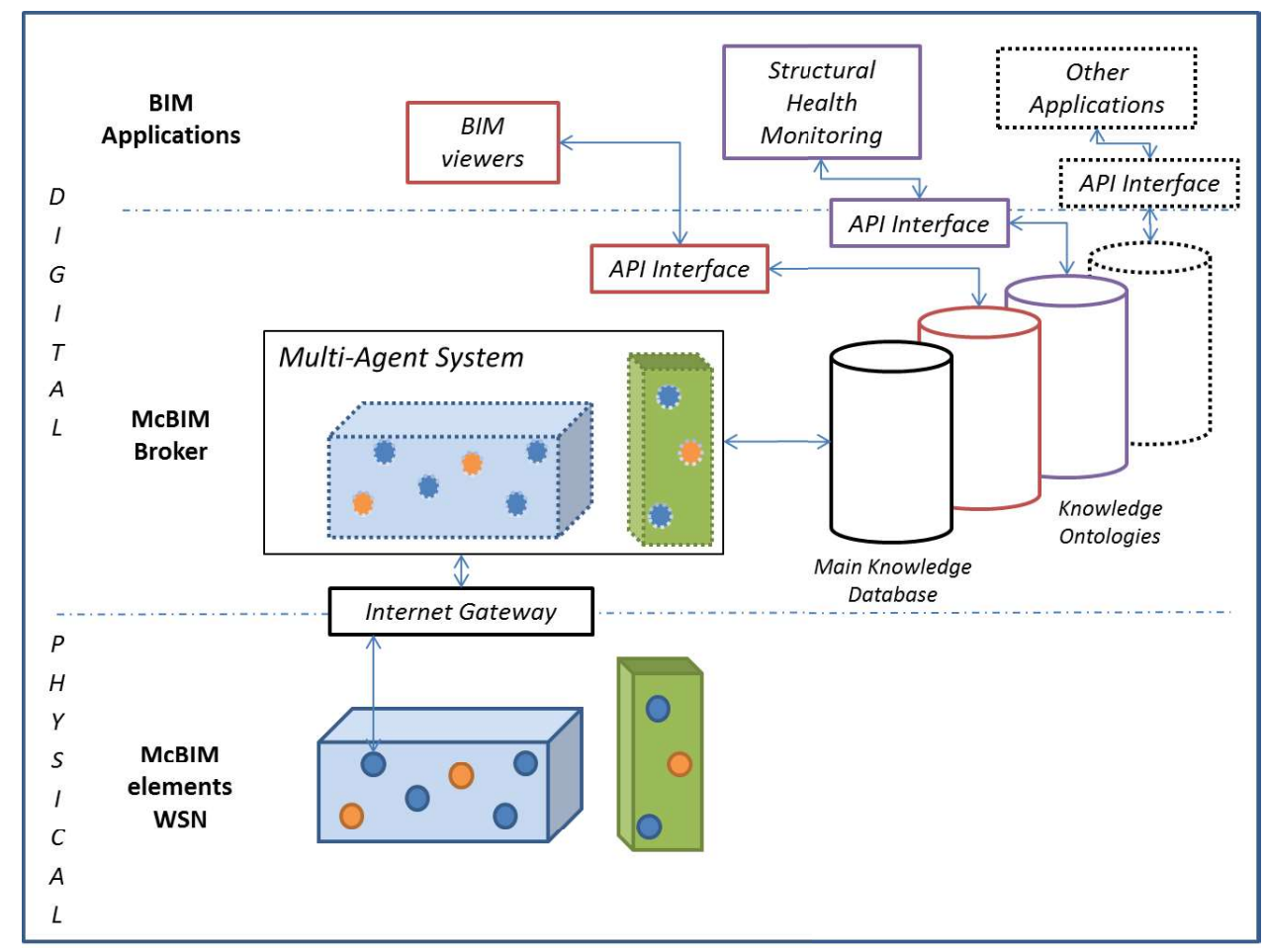

Figure 3 McBIM overview

In this paper, mainly dedicated to the physical part of the McBIM project, we just introduce the overall architecture in which the communicating concrete could be used (see Figure 3). Another challenge of the project is the realization of the link between physical and digital elements contained in a structure called "Broker". The McBIM Broker is a container of software agents, each of them representing a communicating concrete element in the digital world. As a result, agents in the digital world are simple twins of the real concrete elements. These agents are stored in a distant server where they can have access to more computation power and memory. data coming from the real communicating concrete elements are then gathered in data repositories organized using several specific ontologies. External BIM applications can then access the McBIM broker via IFC-based API interfaces. The McBIM broker is still to be design.

\subsection{Proposed network architecture}

According to the diverse targeted use cases and applications - especially for structural health monitoring (SHM) of concrete structure - a wireless mesh network architecture was proposed to design the communicating reinforced concrete. The first 
implementations and tests in the air were performed regarding wireless measurement. The main obtained results are available in [4] and [5].

The proposed hardware implementation of the communicating reinforced concrete is based on the deployment of a two-level wireless mesh network as presented in Figure 4. This network consists of the interconnection of two kinds of node: the communicating nodes $(\mathrm{CN})$ and the sensing nodes $(\mathrm{SN})$. The sensing nodes must sense relevant parameters from the monitored structure and transmit though a unidirectional communication the measured data to the communicating nodes. They become inaccessible once deployed in the concrete. Thus, they must be fully wireless, low power, simple, robust and autonomous for the entire lifespan of the structure (i.e., for decades). The communicating nodes must recover the data transmitted by the sensing nodes in their neighborhood, process, store and exchange with the other communicating nodes -though a bidirectional communication- this data, as well as connect the physical and digital worlds through the Internet to update the building information modeling (BIM).

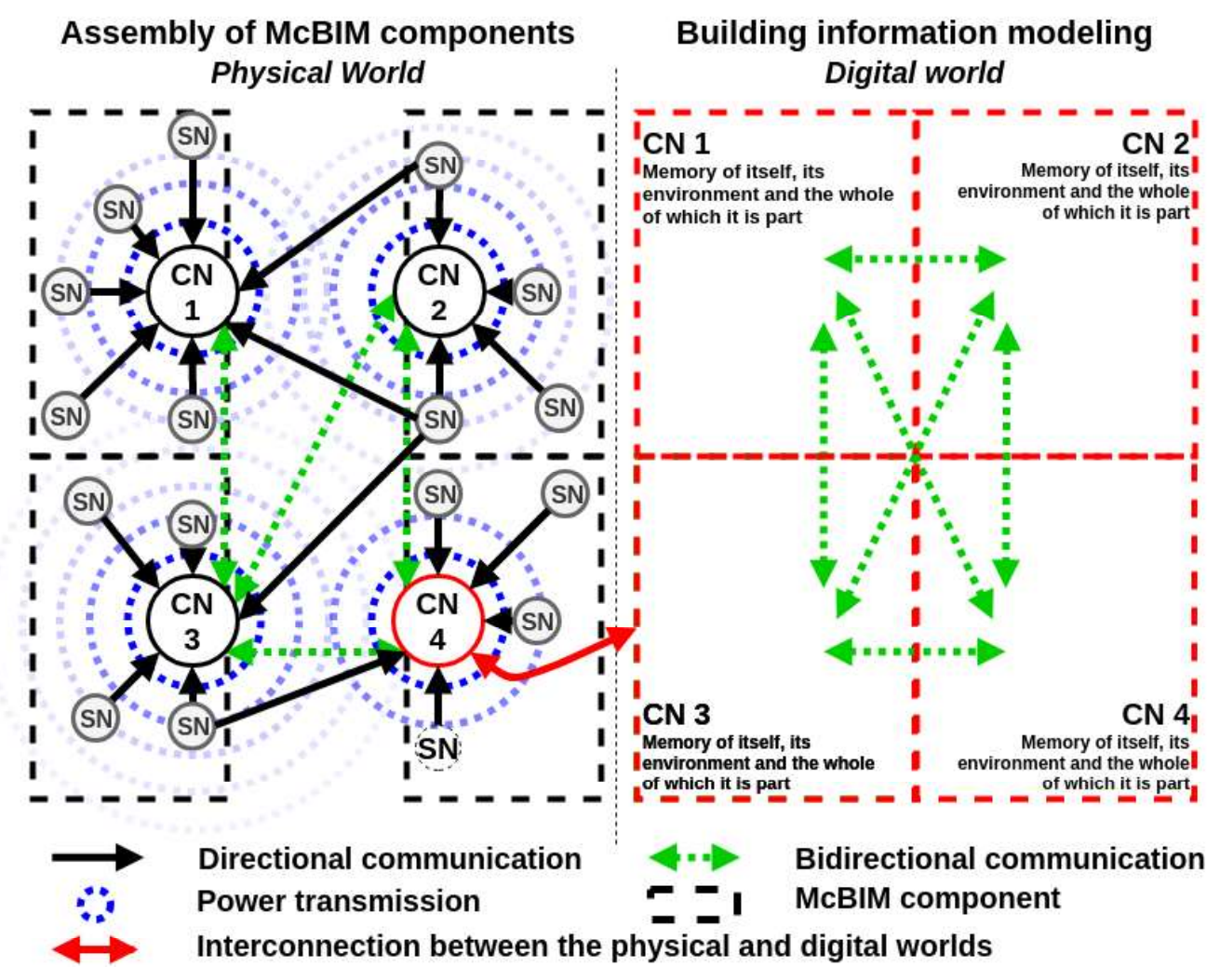

Figure 4 Schematic diagram of the architecture of the proposed network

\section{Development of a Battery-free sensing node device}

\subsection{First implementation and test}

Before embedding this network in the reinforced concrete, it has been developed for tests in the air. First, a sensing node prototype presented in Figure 5 and in $[4,5]$ has 
been designed. It is wireless, battery-free and wirelessly powered through a far-field wireless power transmission (WPT) system in order to be autonomous during the entire lifetime of the concrete element. It is composed of (i) a rectenna to scavenge the radiofrequency (RF) power generated to this aim and convert it in DC power; (ii) a power management unit (PMU) to manage the power provided by the rectenna and store it in a supercapacitor, (iii) a supercapacitor to store enough energy to power the active components of the prototype, (iv) a temperature and relative humidity sensor to measure relevant parameters from the environment, (v) a microcontroller (MCU) to drive the sensor and the transceiver, and (vi) a LoRa transceiver to transmit the measured data to the communicating node. This prototype is not yet miniaturized. Currently, the communicating node is not implemented but a RF power generator (for the wireless power transmission) and a LoRa gateway (for the communication) achieve its functions and can be considered as the communicating node.
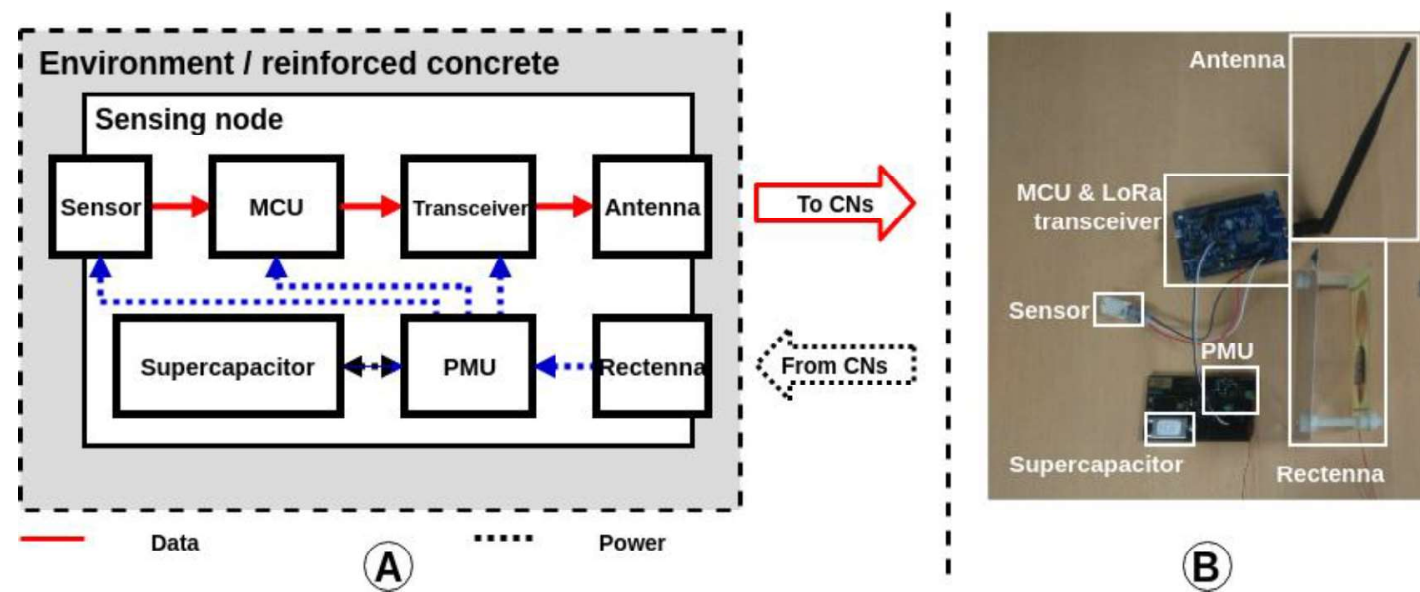

Figure 5 A) Schematic diagram of the architecture of the battery-free wireless sensing node. B) Photograph of an implemented prototype of sensing node.

The tests carried out in the air allows to have a proof-of-concept of a LoRaWAN battery-free and wirelessly powered wireless sensing node designed to structural health monitoring applications. This prototype can be powered over few meters (theoretically until $5.5 \mathrm{~m}$ for the current version), communicate measured temperature and relative humidity data thanks to the LoRa technology and LoRaWAN protocol over kilometers and its periodicity of measurement and transmission can be controlled by tuning the generated RF power used by the wireless power transmission system.

\subsection{Battery-Free Sensing Nodes for Structural Health Monitoring Applications}

Since some years, implementations of battery-free sensing nodes have been proposed to answer the needs of Cyber-Physical Systems and/or SHM applications. Several are based on the LoRa technology and LoRaWAN protocol and used ambient energy harvesting (e.g. mechanical [6], solar [7], etc.) or backscattering [8] techniques. They are dependent of the availability of these ambient energy sources, which fluctuate continuously and are not necessary available in all kind of environments (e.g. into concrete). However, although demonstrated theoretically in [9], no implementation of LoRaWAN SN powered by a far-field WPT approach was provided until [5]. Contrary to near-field WPT solutions, e.g. reported in [10], far-field WPT approach permits larger 
distances of use. Few projects offer solutions for far-field wirelessly powered SNs, e.g. $[11,12]$ which are based on the Bluetooth communication technology.

\section{Data collection protocols in the network of communicating concretes}

The first challenge concerning the network of communicating concretes is to ensure the long-term service, namely, the energy conservation in WSN for each McBIM element during the manufacturing/logistic phase and also with McBIM elements composition during construction and exploitation phases. Here, we focus on the related research for the challenges in the physical part related to the minimization of the energy consumption of data collection in WSN along its whole lifecycle.

Aggregation techniques are widely used for data collection in WSN, as described in [13]. It needs three elements to be effective: an adapted structure of the network (for routing the data), aggregation functions (for fusing the data) and the data representation (for exploiting the data). Second and third elements are problems mainly depending on the application case. "Aggregation functions" depends on the service which is offered by the WSN and "Data representation" consists of the final exploitation of data by user (can be considered as an external problem for the WSN). In this work, we just focus on the preliminary step for aggregation: the WSN structure.

Table 1 presents a selection of protocols based on different types of structures: $(\mathrm{Ch})$ for Chain-based, $(\mathrm{Cl})$ for Cluster-Based and (Tr) for Tree-Based. For our application purposed, different performance metrics for the data aggregation routing protocols have been chosen and are described as follows: energy efficiency represents the algorithm's efficiency for data collection; Maintaining cost represents the energy consumption for reconstruction of the architecture; Resilience to link failure indicates the robustness of the network facing the loss of link when a node dies; Scalability stands for the capability to scale the scenario.

Table 1 SUMMARY OF DATA AGGREGATION PROTOCOLS IN WSN

\begin{tabular}{|c|c|c|c|c|}
\hline $\begin{array}{c}\text { Protocol name } \\
\text { (Type of structure) }\end{array}$ & $\begin{array}{c}\text { Energy } \\
\text { efficiency }\end{array}$ & $\begin{array}{c}\text { Maintaining } \\
\text { cost }\end{array}$ & $\begin{array}{c}\text { Resilience to } \\
\text { link failure }\end{array}$ & Scalability \\
\hline PEGASIS $(\mathrm{Ch})$ & - & -- & - & -- \\
\hline CCM $(\mathrm{Ch})$ & + & - & - & -- \\
\hline CRBCC $(\mathrm{Ch})$ & + & + & + & - \\
\hline BCBRP $(\mathrm{Ch})$ & - & -- & ++ & -- \\
\hline LEACH $(\mathrm{Cl})$ & -- & + & + & -- \\
\hline TL-LEACH $(\mathrm{Cl})$ & - & + & + & - \\
\hline HEED $(\mathrm{Cl})$ & + & - & + & + \\
\hline DWEHC $(\mathrm{Cl})$ & ++ & - & ++ & + \\
\hline EADAT $(\mathrm{Tr})$ & - & -- & - & + \\
\hline PEDAP $(\mathrm{Tr})$ & ++ & + & - & + \\
\hline TAG $(\mathrm{Tr})$ & + & ++ & - & + \\
\hline
\end{tabular}

The plus and minus represent the strength and weakness of these routing protocols. This table can then be used to select suitable algorithms for our application. 
Due to the difference of the communicating concrete behaviors over the three phases, we aim to find the most suitable approach for each phase. As mentioned above, the communicating concrete mainly monitors its status during the manufacturing. Therefore, a simple and efficient data aggregation protocol will be preferred. Table 1 shows that DWEHC and PEDAP have better energy efficiency than the others. Meanwhile, DWEHC provides a high resilience to link failures, PEDAP has a low maintaining cost. In this case, both are considered relevant for the manufacturing phase.

For construction, the problems are not only the minimization of energy inside each beam, but also the re-organization of network between concrete beams. Thus, a data aggregation technique favoring dynamic and low maintaining cost network should be privileged. Although the cluster-based approach like DWEHC provides a good scalability and high energy efficiency, its high maintaining costs are not suitable for this phase. In contrast, PEDAP has a low maintaining cost with a good scalability and high energy efficiency and thus may be a good choice for this phase. Although TAG provides lower energy efficiency than PEDAP, its scalability and lower maintaining cost may also give a good result.

After the construction, a large network will be completed and used for the exploitation phase. As a result, a robust and low maintaining cost protocol may be the most efficient solution. Therefore, a protocol with high energy efficiency, high tolerance to link failure and low maintaining cost will be the best choice. From the table 1, CRBCC may have good result, but it cannot be used in the context of communicating material, because all the nodes should directly communicate with the BS for this protocol to work. The high energy efficiency and resilience to link failure of DWEHC may lead a good result for periodical monitoring. In addition, different physical phenomena will be monitored, such as temperature, humidity, etc. The data-centric treebased routing protocol TAG will also be considered.

\section{Conclusion and perspectives}

This paper describes a research project named McBIM. Its main objective is the design of communicating concretes interacting during the overall lifecycle with BIM applications. Main physical challenges relative to hardware design of battery-free sensing node and control of the network of nodes are defined and some issues are addressed. The first tests and results on our sensing node device power with RF harvesting seem to be promising. This first step allows the design of a system which could be used during several decades. Another energetic challenge is the optimization of the network structure. A synthesis of existing solutions and protocols is proposed and discussed relatively to each phase of the McBIM concrete lifecycle. These protocols will be tested in a real platform in order to validate which protocol should be implemented for each specific phase.

As discussed in section 2 (see Figure 3), this project also includes digital challenges. In this part, the main goal is the physical data exploitation by a Multi-Agent System in order to produce knowledge organized in repositories based on specific and applicationrelated ontologies. Each of these repositories will be tested in interaction with its corresponding BIM application. Considering the exploitation phase of a building, the 
future McBIM broker could successfully interact with any kind of monitoring application, especially Structural Health Monitoring.

\section{Acknowlegements}

This work was supported by the French National Research Agency (ANR) through the McBIM Project (Communicating Material at the disposal of the Building Information Modeling), under Grant ANR-17-CE10-0014. The authors thank the ANR for its support.

\section{REFERENCES}

1. S. Kubler, K. Främling, W. Derigent, and M. J. Rabita, "Premiers travaux relatifs au concept de matière communicante: Processus de dissémination des informations relatives au produit," PHD Thesis, Université de Lorraine, 2012.

2. K. Mekki, "Gestion de l'information embarquée dans des matériaux communicants à l'aide de protocoles de réseaux de capteurs," PHD Thesis, Université de Lorraine, 2016.

3. "ANR Project McBIM," 2017. [Online]. Available: http://mcbim.cran.univ-lorraine.fr/Members.

4. Loubet, G., Takacs, A., and Dragomirescu, D.. 2018. "Towards the Design of Wireless Communicating Reinforced Concrete," IEEE Access, 6: 75002-75014.

5. Loubet, G., Takacs, A., Gardner, E., De Luca, A., Udrea, F., and Dragomirescu. D.. 2019. "LoRaWAN Battery-Free Wireless Sensors Network Designed for Structural Health Monitoring in the Construction Domain," MDPI Sensors, 19 (7), 1510, 1-26.

6. Francesco, C.B. Mezzetti, and F. Cottone. 2016. "Vibrations powered LoRa sensor: An electromechanical energy harvester working on a real bridge," Proceedings of the IEEE Sensors, Orlando, Florida, USA, 30 October - 03 November 2016, 1-3.

7. T. Polonelli, D. Brunelli, M. Guermandi, and L. Benini. 2018. "An accurate low-cost Crackmeter with LoRaWAN communication and energy harvesting capability," Proceedings of the IEEE 23rd International Conference on Emerging Technologies and Factory Automation (ETFA), Torino, Italy, 4-7 September 2018, 1:671-676.

8. W.K. Lee, M.J. Schubert, B.Y. Ooi, and S.J.Q. Ho. 2018. "Multi-Source Energy Harvesting and Storage for Floating Wireless Sensor Network Nodes With Long Range Communication Capability," IEEE Transactions Applications, 54(3):2606-2615.

9. S. Tjukovs, J. Eidaks, and D. Pikulins. 2018. "Experimental Verification of Wireless Power Transfer Ability to Sustain the Operation of LoRaWAN Based Wireless Sensor Node," Proceedings of the IEEE Advances in Wireless and Optical Communications (RTUWO), Riga, Latvia, 15-16 November 2018, 83-88.

10. S. Johann, C. Strangfeld, M. Müller, B. Mieller, and M. Bartholmai. 2017. "RFID sensor systems embedded in concrete-requirements for long-term operation," Materials Today: Proceedings, 4(5):5827-5832.

11. R. La Rosa, C. Trigona, G. Zoppi, C.A. Di Carlo, L. Di Donato, and G. Sorbello. 2018. "RF energy scavenger for battery-free Wireless Sensor Nodes," Proceedings of the IEEE International Instrumentation and Measurement Technology Conference (I2MTC), Houston, Texas, USA, 14-17 May 2018, 1-5.

12. Y. Syed, B.G. Hegde, T.V. Prabhakar, M. Manjunath, and K.J. Vinoy. 2016. "RF energy harvesting chip powered sensor node," Proceedings of the IEEE International Conference on Electronics, Circuits and Systems (ICECS), Monte Carlo, Monaco, 11-14 December 2016, 748-751.

13. E. Fasolo, M. Rossi, J. Widmer, and M. Zorzi, "In-Network Aggregation Techniques for Wireless Sensor Networks: a Survey," IEEE Wireless Communications, no. April, pp. 70-87, 2007. 


\section{DEStech Publications, Inc.}

\section{CONTRIBUTING AUTHOR COPYRIGHT RELEASE FORM}

As author of the chapter/contribution titled Materials communicating with the BIM: aims and first results of the McBIM project, to appear in the Proceedings of Structural Health Monitoring 2019, I hereby agree to the following:

1. To grant to DEStech Publications, Inc., 439 North Duke Street, Lancaster, PA, 17602, copyright of the above named chapter/contribution (for U.S.

Government employees to the extent transferable), in print, electronic, and online formats. However, the undersigned reserve the following:

a. All proprietary rights other than copyright, such as patent rights.

b. The right to use all or part of this article in future works.

DEStech Publications thereby retains full and exclusive right to publish, market, and sell this material in any and all editions, in the English language or otherwise.

1 I warrant to DEStech Publications, Inc., that I am the (an) author of the above-named chapter/contribution and that I am the (a) copyright holder of the above-named chapter/contribution granted to DEStech Publications, Inc.

2 I warrant that, where necessary and required, I have obtained written permission for the use of any and all copyrighted materials used in the abovenamed chapter/contribution. I understand that I am responsible for all costs of gaining written permission for use of copyrighted materials.

3 I agree to assume full liability to DEStech Publications, Inc. and its licensee, and to hold DEStech Publications, Inc. harmless for any claim or suit filed against DEStech Publications, Inc. for violation of copyrighted material used in the abovenamed contribution.

Please sign and date this form and retain a copy for your records. Please include original form with your chapter/paper.

Thank you for your cooperation.

Please print name: William DERIGENT

Signed:

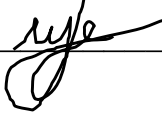

Dated: $31 / 05 / 2019$

439 NORTH DUKE STREET • LANCASTER, PENNSYLVANIA 17602-4967, U.S.A. Toll Free: (866) 401-4337 • Tel: (717) 290-1660 • Fax: (717) 509-6100 E-mail: info@destechpub.com • Internet address: www.destechpub.com 